\title{
Diabetes and cancer: A critical appraisal of the pathogenetic and therapeutic links (Review)
}

\author{
VALERIO GRISTINA, MARIA GRAZIA CUPRI, MARTINA TORCHIO, \\ CLAUDIO MEZZOGORI, LAURA CACCIABUE and MARCO DANOVA
}

Internal Medicine and Medical Oncology, Ospedale Civile, Vigevano, Azienda Ospedaliera di Pavia 19, I-27029, Italy

Received November 16, 2014; Accepted November 19, 2014

DOI: $10.3892 /$ br.2014.399

\begin{abstract}
Diabetes and cancer represent two common, multifactorial, chronic and potentially fatal diseases, not infrequently co-diagnosed in the same patient. Epidemiological data demonstrate significant increases of the cancer incidence in patients with obesity and diabetes, which is more evident for certain site-specific cancers. Although there is increasing evidence that strongly indicates an augmented risk of cancer in diabetic patients, several confounding factors complicate the ability to precisely assess the risk. Mainly in insulin-resistant states (such as in type 2 diabetes mellitus and in metabolic syndrome), direct associations between obesity-related hyperinsulinemia and increasing circulating insulin-like growth factor-1 (IGF-1) levels have been implicated as key factors in the mechanisms involved in carcinogenesis. Whilst anti-diabetic drugs can increase the cancer risk, anti-proliferative drugs may cause diabetes or aggravate pre-existing diabetes. Additionally, an increasing number of targeted anti-cancer therapies may interfere with the pathways shared by IGF-1 and insulin receptors, showing a adverse effect on glucose metabolism through various mechanisms. Although there is a requirement for large-scale randomized evidence, the present review summarizes the majority of the epidemiological association studies between diabetes and various types of cancer, discussing the pathophysiological mechanisms that may be involved in promoting carcinogenesis in diabetes and the potential impact of different anti-diabetic therapies on cancer risk.
\end{abstract}

\section{Contents}

1. Introduction

2. Diabetes and risk ratio to cancer

3. Similar pathogenic pathways shared by diabetes and cancer

Correspondence to: Dr Marco Danova, Internal Medicine and Medical Oncology, Ospedale Civile di Vigevano, Azienda Ospedaliera di Pavia, Corso Milano 19, I-27029, Vigevano, Italy E-mail:marco_danova@ospedali.pavia.it

Key words: diabetes, cancer, risk, insulin-resistant states, anti-diabetic medications, anti-cancer drugs
4. Incidence of different types of cancer among diabetic patients

5. Diabetes, cancer and therapies

6. Conclusion

\section{Introduction}

The association between diabetes and cancer, two common diseases with a significant impact on health worldwide affecting developed and developing countries, was suggested prior to the 21 st century, indicating key clinical, biochemical and metabolic commonalities. Type 2 diabetes mellitus (T2DM), the seventh leading cause of mortality, is increasing in prevalence, and strongly influencing the morbidity and mortality rates. Cancer is the second leading cause of mortality, and is also increasing in prevalence (1). With regards to the global epidemic of diabetes, a moderate increase in the cancer risk may translate into a significant socioeconomic burden (2).

Several cohort studies and meta-analyses have strongly demonstrated that the T2DM and insulin-resistant states (such as in metabolic syndrome) are independent risk factors for the development of several types of cancer (1,3-5). There is also increasing evidence confirming that these two high-impact diseases share factors that influence their development and progression, significantly modifying the outcome of the other. In addition, it is currently believed that diabetes is a marker of elevated cancer risk due to changes in underlying metabolic conditions, such as insulin resistance or hyperinsulinemia. Alternatively, the multidrug therapy required for the treatment of diabetes and cancer may complicate this association (3).

However, in addition to the non-randomized nature of the studies taken into account, certain published data are conflicting, requiring reinterpretation as DM is not a single disease, but a group of metabolic disorders characterized by a series of potential confounding factors (obesity, varying levels of metabolic control, profiles of anti-diabetic treatment and possible chronic complications or comorbidities) that may influence the association between diabetes and cancer (6).

The purpose of the present review is to further elucidate the association between diabetes and cancer incidence (overall and specific cancers), examine the potential underlying pathogenic mechanisms involved and discuss the possible link 
between glucose-lowering medications, antiproliferative drugs and these two chronic diseases.

\section{Diabetes and risk ratio to cancer}

Common non-adjustable (age, gender and race/ethnicity) and adjustable (weight, diet, physical activity, tobacco and alcohol use) risk factors determine the development of cancer and T2DM.

In economically-developed countries, $78 \%$ of all newly diagnosed cancers occur among individuals aged $\geq 55$ years. Diabetes is also increasingly common with age (prevalence increases to $23.8 \%$ in those $\geq 60$ years). Whereas certain cancers are gender-specific (including cervix, uterine, testicular and prostate), overall, cancer occurs more frequently in males who have a moderately higher age-adjusted risk of diabetes compared to females. Additionally, genetic, socioeconomic, lifestyle and other environmental factors are believed to contribute to the ethnic variability in the cancer incidence, whereas T2DM disproportionately affects various populations. Overweight and obese individuals have a higher risk for numerous types of cancer and show a strong association with insulin resistance and T2DM incidence compared to individuals whose body mass index (BMI) is within the normal range $\left(18.5\right.$ to $\left.<25 \mathrm{~kg} / \mathrm{m}^{2}\right)$. The majority of studies indicate that diets that are low in red and processed meats and higher in vegetables, fruits and whole grains are associated with a lower cancer risk and may protect against T2DM, possibly due to improved insulin sensitivity and glycemic control $(4,5)$. Evidence from observational epidemiological studies shows that physical activity may help prevent the incidence of cancer, T2DM and their complications, whereas tobacco smoking and excess alcohol consumption have adverse effect on diabetes-related health outcomes and are independent risk factors for numerous types of cancer $(4,5,7,8)$.

Several cohort studies and meta-analyses confirm that diabetes has been consistently associated with an increased risk for broad variety of solid and hematological malignancies $(9,10)$. While for prostate cancer a reduced incidence has been reported in the diabetic population and there is no proven link between lung neoplasms and DM, there is evidence for the development and the progression of malignancies among diabetics for liver, pancreas, colorectal, kidney, bladder, endometrial and breast cancers (9).

When hyperinsulinemia acts as a critical link between the observed increased cancer risk and diabetic patients, it is reasonable to assume that the large majority of tumors observed in diabetic patients occurred in T2DM, considering the 1:10 ratio between type $1 \mathrm{DM}$ (T1DM) and T2DM and postulating that cancer is mainly a disease of the older population in which T1DM is less frequent. Conversely, although there are limited studies, Zendehdel et al (11) reported that T1DM can increase the incidence risk $\leq 20 \%$ for gastric, endometrial and cervical cancer in the general Swedish population. T1DM appeared to be correlated with a moderate excess cancer risk overall, and risks of specific types of cancer that differ from those associated with T2DM (12). To the best of our knowledge, the exact values of estimated risk, however, may vary due to study design, the impact of confounding factors and ethnic differences, including genetic susceptibility, life-style behaviors, specific environmental exposures and varying biological effects of diabetes across populations.

\section{Similar pathogenic pathways shared by diabetes and cancer}

In diabetic patients, carcinogenesis may be favoured either by general mechanisms promoting cancer initiation or progression in any organ, or by site-specific mechanisms affecting a particular organ. There appears to be a critical interaction between hyperglycemia, hyperinsulinemia, peripheral insulin-resistance and central adiposity, creating a low-grade chronic inflammatory state $(1,4,5,13)$.

Insulin, due to its known mitogenic effects in addition to its metabolic functions, has been strongly indicated as a critical mediator in the complex mechanisms involved in carcinogenesis. Although insulin and insulin-like growth factor-1 (IGF-1) share the affinity to the insulin receptor (IR) and IGF-1 receptor (IGF-1R) due to similar structural homology, it is of note that the affinity of insulin for the IR is $>1,000$-fold greater compared to for IGF-1R (the A isoform of the IR, commonly expressed in malignant cells, stimulates insulin-mediated mitogenesis even in cells deficient in IGF-1 receptors). In addition, insulin may also indirectly promote cancer development via IGF-1, decreasing the hepatic production of the IGF-binding protein-1 [and sex hormone-binding globulin (SHBG)], which may increase the circulating levels of free bioactive IGF-1 (and bioavailable estrogen and testosterone), potentially enhancing the promotion and progression of numerous types of cancer cell (10).

Furthermore, obesity-related hyperinsulinemia may finally upregulate the insulin mitogenic compared to the insulin metabolic pathway. Insulin resistant states, including obesity, metabolic syndrome and T2DM, cause impairment of downstream GLUT4 translocation by disruption of IR substrate-1 (IRS-1) associated phosphoinositide 3-kinase (PI3K) signaling in the metabolic pathway of insulin. By contrast, insulin stimulates cellular growth and protein synthesis through the protein kinase B system and activation of mammalian target of rapamycin (mTOR). Consequently, in hyperinsulinemia, abnormal IRS-1 phosphorylation from mTOR overactivation generates a negative feedback loop that attenuates the metabolic pathway, whereas IRS-2 expression by insulin phosphorylation leads to increased extracellular signal-regulated kinase activation, a mitogen-activated protein kinase pathway, moving towards the mitogen pathway and enhancing cellular growth and survival. In this subset of patients, hyperglycemia may also have a direct effect, since cancer cells are dependent on glycolysis for energy $(4,10,14)$.

Beside the direct insulin effects, T2DM and/or the associated obesity may trigger other pathways resulting in malignant progression. Central adiposity highly correlates with insulin resistance and is a key factor in metabolic syndrome. Therefore, adipose tissue-derived cytokines [resistin, tumor necrosis factor (TNF)- $\alpha$ and interleukin (IL)-6], free fatty acids and other vascular factors released from the visceral compartment, which work in opposition to adiponectin, stimulating insulin sensitivity, have been proven to increase the cancer risk by perpetuating low-grade inflammation, insulin resistance and glucose intolerance that collectively generate an eligible environment for cancer development and progression. In particular, 
obese patients have been shown to experience poorer outcomes from treatment: Significantly longer surgical times undergoing surgical resection, reduced effectiveness of radiotherapy in certain instances or possible under-dosage of chemotherapeutic agents owing to concerns that large doses calculated based upon body weight would cause excess toxicity (5).

Emerging data indicate that persistent inflammation promotes genetic instability and chronic inflammation, putting susceptible cells at risk toward malignant transformation, and this is associated with an increased cancer risk. In poorly-controlled diabetes, the deregulated metabolism causes a long-term pro-inflammatory state characterized by increased levels of IL-6, TNF- $\alpha$, C-reactive protein and other markers of chronic inflammation. Chronic inflammation and chronic oxidative stress go together. While reactive oxygen species (ROS) can initiate carcinogenesis by damaging proteins and DNA, chronic inflammation is associated with high levels of TNF- $\alpha$ strongly activating nuclear factor- $\kappa \mathrm{B}$, which is involved in the proliferation and survival of malignant cells, and also mediates responses to chemotherapeutic agents (15).

\section{Incidence of different types of cancer among diabetic patients}

According to several meta-analyses, an increased frequency of malignancies in diabetic patients has been attributed to various general and local mechanisms $(1,3,4,10,12)$.

The strongest association between DM and augmented cancer risk is with pancreatic and liver cancer, two main target organs for insulin metabolism involved in the metabolic derangements typical of diabetes.

The hepatocellular carcinoma (HCC) incidence is higher in those with T2DM in the two genders, with a greater risk in males and in those with concomitant hepatitis $\mathrm{C}$ virus (HCV) infection: As the majority of epidemiological studies indicate a 2-3-fold increase in liver cancer in diabetic patients, it is feasible that diabetes may also act synergistically with other well-established risk factors for HCC, including hepatitis $\mathrm{B}$ virus and $\mathrm{HCV}$ infection, non-alcoholic fatty liver disease (also considered as hepatic manifestation of diabetes and metabolic syndrome), non-alcoholic steatohepatitis and chronic alcohol consumption leading to steatosis and cirrhosis $(1,3,16)$. In particular, the mitogenic pathway of insulin is unlikely to be involved considering that healthy liver cells, due to insulin secretion bursts in the portal circulatory system, are physiologically exposed to higher insulin concentrations compared to other peripheral tissues (10), above all in the insulin-resistant hyperinsulinemic type 2 states (whereas in insulin-deficient T1DM individuals treated with exogenous insulin, the liver is exposed to the same insulin levels as the other organs). Additionally, in a large nested case-control study of liver cancer in patients with T2DM, it was shown that the use of any anti-diabetic medication was not associated favorably or adversely with liver carcinogenesis, although use of only metformin was associated with a consistent, yet non-statistically significant, protective effect in comparison to non-use (17). In conclusion, the exact mechanisms underlying a supposed oxidative stress condition remain controversial, and the increased liver cancer incidence is well-documented among diabetic patients.
The majority of earlier meta-analyses address diabetes as an independent risk factor for pancreatic cancer (18-20). A recent study shows that a $30 \%$ excess risk persists more than two decades following the diabetes diagnosis, thus supporting a causal role of diabetes in pancreatic carcinogenesis, and also stating that oral anti-diabetics may decrease the risk, whereas insulin exhibits an inconsistent duration-risk association (18). However, these studies are misleading as they do not distinguish between pre-existing and new-onset diabetes (possibly due to undiagnosed pancreatic cancer causing a functional damage). Additionally, given the negative correlation of the DM duration with the pancreatic cancer risk (higher risk of cancer among the studies was found within the first year of follow-up and subsequently it gradually decreased), the controversy regarding the causal role of diabetes has increased, generating the theory of reverse causality in which pancreatic cancer induced a diabetic state regardless of smoking status or BMI. However, when epidemiological evidence suggests a reciprocal link between diabetes and pancreatic cancer, the temporal patterns of association and the causative link between the two were not clear (20). Although additional larger studies are required to further examine the potential confounding effect of smoking and obesity on the association between diabetes and pancreatic cancer, the unclear biological mechanisms underlying this association indicate hyperinsulinemia as a major key factor since exocrine pancreatic cells are exposed to extremely high insulin concentrations due to the common blood supply with the adjacent insulin-secreting islets.

Studies concerning other types of cancer are limited compared to for liver and pancreatic studies, and the increases in relative risk (RR) are not as statistically significant. However, particularly considering the growing prevalence of the two diseases in the general population, the increased risk is clinically considerable.

T2DM has been associated with an increased risk of colorectal adenomas and adenocarcinomas in females and males, and also with an increased risk of short- and long-term mortality (21), suggesting that common features among diabetics (such as slower bowel transit and constipation, as well as increased levels of IGF-1, insulin and glycated haemoglobin) may lead to prolonged exposure of colon mucosa to toxins and potential carcinogens. In addition, although the requirement for more studies with longer duration of insulin-based therapy, meta-analyses of observational studies recently indicated a strong association between insulin therapy and colorectal cancer (CRC), with a $69 \%$ increase in the risk of CRC $(22,23)$.

Breast and endometrial cancer patients are also more likely to exhibit a history of diabetes, strongly considering that hyperinsulinemia is associated with excessive ovarian androgen secretion and decreased levels of circulating sex hormone-binding protein, which collectively lead to higher concentrations of bioactive estrogens; known risk factors for malignancies of female reproductive organs. In addition, DM has been associated with adverse outcomes in breast cancer throughout its full course, from treatment (affecting the choice of treatment) to mortality rates (24).

The increased incidence and mortality for renal cancer among diabetic patients have been attributed to general mechanisms (hyperinsulinemia and obesity) and specific frequent co-morbidities for diabetes [hypertension and end-stage renal 
disease (ESRD)]. Liver, lung and, in particular, urothelial cancers are associated with the impaired excretory renal function in chronic kidney disease (the lesser stage of ESRD), resulting in higher circulating levels of carcinogenic toxins and immune inhibition.

Individuals with DM also exhibit a moderate increase in the risk of bladder cancer, particularly in males $(25,26)$, cholangiocarcinoma (increased formation of biliary stones), esophageal carcinoma (mainly adenocarcinoma, due to delayed gastric emptying and subsequent dyspepsia, which are more common among diabetic patients and simultaneously able to lead to Barrett's esophagus) and gastric cancer (27).

Hematological malignancies also appear to be associated with diabetes. Meta-analyses report a higher risk of non-Hodgkin lymphoma (particularly peripheral T-cell lymphoma), myeloma and leukemia $(1,3,10)$.

There are also studies contradicting an association between diabetes and cancer, specifically for ovarian, lung and prostate malignancies. In prostate cancer particularly, T2DM appears to have a protective effect and a temporal association, similar to pancreatic cancer, with the protective effect occurring later in T2DM. The $16 \%$ average decreased risk of developing prostate cancer should be most likely associated with the dihydrotestosterone, testosterone and SHBG haematic levels, inversely correlated with circulating insulin. However, other metabolic and hormonal conditions have been indicated, including altered insulin and leptin concentrations, the diffuse use of medicine, such as statins and metformin, and changes in diet and lifestyle in order to correct diabetes. Alternatively, regarding the lung, a Taiwanese study strengthens the link between diabetes and lung cancer, which is more evident in the age of $<40$ years and assumes that none of the anti-diabetic medications, including insulin, affect the risk of lung cancer (28).

\section{Diabetes, cancer and therapies}

Anti-diabetic drugs and cancer risk. With regards to the diabetes and cancer association, the most direct concern for patients, as well as clinicians, is that various treatment arms appear to affect the risk of cancer. By contrast, a recently emerging issue is the possible adverse effect on glucose metabolism of certain anti-proliferative therapies.

At present, improved glucose control is one of the main goals of effective diabetes management, which aims to minimize morbidity and mortality by reducing diabetes-associated complications. Since management of T2DM is changing over time and is being adjusted to each patient individually, the potential role of the anti-diabetic drugs in cancer is unclear, but most likely minor. Epidemiological studies regarding this are difficult to interpret and often not conclusive.

Due to the proposed mechanistic association of endogenous hyperinsulinemia with cancer growth and promotion, there is a concern that exogenously administered insulin may amplify the cancer development process. Notably, while the physiological pattern of insulin secretion to the portal vein includes degradation and utilization of a significant proportion of the hormone in the liver, exogenous administration allows higher availability of insulin to the peripheral tissues (12). However, a Chinese cohort study demonstrated that there was no excess risk of overall cancer in T2DM patients treated with human insulin, regardless of an increased overall and cancer mortality among insulin users (29).

The three major oral anti-diabetic drug families [sulphonylureas, biguanides and thiazolidinediones (TZDs)] have different mechanisms of action. When hyperinsulinemia is considered as a key factor in augmenting cancer risk and progression among diabetic patients, it is reasonable to expect that these drugs will have a different effect on the association between diabetes and cancer.

Sulphonylureas are secretagogues stimulating endogenous insulin secretion and causing hyperinsulinemia, whereas the other two categories of compounds are insulin sensitizers that make the tissues more responsive to insulin, therefore decreasing hyperinsulinemia. Alternatively, in terms of the ongoing debate on the role of hyperinsulinemia in tumorgenesis, there is a growing concern that drugs, such as insulin secretagogues (sulphonylureas), may represent a plausible risk factor, showing a close association with a higher cancer incidence and mortality (21).

The biguanide metformin, a first line and most commonly used drug in diabetes management, has recently attracted increasing attention as strong evidence has reported its anti-neoplastic properties. The most recent meta-analyses demonstrated that metformin was associated with a $39 \%$ lower cancer risk, as compared to reference anti-diabetic therapies taken together $(6,10,13,19)$. In recent in vitro studies on breast cancer cells, downstream signaling pathway of AMK-aktivated protein kinase (AMPK) comprising protein kinase $\mathrm{B}(\mathrm{AKT})$ and mTOR inhibition, and cyclin D1 and p53 interference, most likely accounts for the antiproliferative effects of metformin. Additionally, this biguanide was shown to repress the process of epithelial-mesenchymal transition, in which cancer cells acquire the metastatic phenotype or to reduce endogenous ROS generation, therefore impeding mutagenesis in somatic cells. With regards to individual types of cancer, metformin use is associated with a significantly lower risk of colorectal, hepatocellular and lung neoplasms, also improving the chemotherapy outcomes in these groups of patients according to prospective clinical trials.

Other classes of compounds used in DM therapy, such as TZDs and incretins, have a less clear link with neoplastic disease. Evidence from a previous meta-analysis and several observational analytical studies reported a potential concern for an increased bladder cancer risk with the use of TZDs (pioglitazone, but not rosiglitazone), particularly with long-term use and large cumulative doses (4). In addition, as obesity is a primary risk factor for adult T2DM, long-term applications of TZDs and insulin are associated with weight gain and possible increase of cancer risk.

Although the quality and relevance of the study are not clear, in March 2013 the Food and Drug Administration released a safety communication stating that the agency was evaluating a new study that suggested an increased risk for precancerous cellular changes (medullary thyroid and pancreatic cancer) in diabetic patients treated with incretin mimetics, such as glucagon-like peptide-1 receptor agonists and dipeptidyl peptidase 4 inhibitors (13).

Anti-cancer drugs and diabetes risk. By contrast, the drugs used to treat cancer may favour diabetes or worsen a condition 
of pre-diabetes. Oncology patients may experience temporary hyperglycaemic states or diabetes following steroid-based medications (administered prior to and during chemotherapy) or due to the specific mechanism of action of anti-cancer drugs. Additionally, glucocorticoids, frequently used at a high dosage for a prophylactic and/or a therapeutic approach during the anti-cancer treatment, may contribute to cause severe insulin resistance, therefore adversely affecting glucose metabolism. In addition to androgen deprivation, the fundamental treatment of prostate cancer increases the risk of diabetes and cardiovascular diseases, which decreases insulin sensitivity and alters the lipid profile, finally resulting in insulin resistance and occasionally, diabetes.

Although the most currently employed targeted anti-cancer molecules do not significantly affect glucose homeostasis, an increasing number of compounds targeting the IGF-1 system and its intracellular pathways are being tested for their anti-cancer effect, despite resulting in altering the insulin-glucose balance. Therefore, numerous efforts have been made to block the well-known role of IGF-1 as a cancer-promoting factor, showing however, a detrimental response on glucose metabolism through various mechanisms, such as inhibiting IGF-1 insulin-mimetic effect, increasing growth hormone $(\mathrm{GH})$ levels due to the lack of IGF-1 feedback or finally, possibly cross-inhibiting the insulin signaling pathway. At present, for the treatment of solid and haematological tumors, the therapeutic strategies targeting the IGF system currently include monoclonal antibodies and protein kinase inhibitors, developed and found to be active in preclinical models and in phase I clinical trials mostly inhibiting the IGF-1R rather than the IGF-1 peptide (10). Specifically, given the complexity of this IGF-1R signaling, tyrosine-kinase inhibitors (TKI), aimed at targeting the mitogenic and survival pathways (such as PI3K, AKT and mTOR), also have effects on the metabolic pathways, causing insulin resistance and altered glucose balance (such as everolimus). Furthermore, when TKIs cause more serious toxicity than that observed with the IGF-1R-blocking antibodies they are associated with less hyperglycaemia, as they possibly do not accumulate in muscle, leaving unaffected IR function on the metabolic process of this tissue. Thus far, useful clinical data concerning all these issues remains insufficient.

\section{Conclusion}

Diabetes is a high-risk factor for various diseases, including cancer. With regards to the current globally expanding diabetes pandemic, there is an increasing requirement to elucidate the pathophysiological links underlying the cancer risk and mortality in the population. The complexity of the different diabetic conditions, the biological diversities of the various types of cancer, the multiplicity of the possible mechanisms involved and a series of confounding factors not considered in the majority of the epidemiological studies complicate the ability to perform studies on the association of these two diseases. Based on the evidence reviewed, plausible mechanisms linking insulin-resistant states and diabetes to the development of cancer have been suggested. Since T2DM may be preventable and is treatable with lifestyle modification and pharmacotherapy, the cancer risk may also be lowered by these strategies. Although anti-diabetic medications have been indicated in increasing the risk of cancer, the observed risks or hazards are less strong (generally $\leq 2$-fold) and do not represent a signal for safety concerns, advocating for current vigilance and future research. As opposed to this, certain glucose-lowering drugs, such as biguanides, are associated with lower risks of specific types of cancer and may even aid as adjunctive therapy in cancer management, while new anti-cancer therapies show an adverse effect on glucose balance. At present, there is currently insufficient evidence to treat ineffectively hyperglycaemic states and to warrant withholding of the use of certain glucose-lowering drugs on the basis of cancer concern. With regards to diabetes, it appears that there is no requirement for changes in clinical practice in terms of anti-diabetic medications. By contrast, there remains the requirement for improved quantifying sub-optimal treatments and outcomes among patients with diabetes that develop cancer (30). Other factors, such as how to optimally adjust chemotherapy doses for increasingly obese patients, have received less attention (31).

In addition, the majority of data linking anti-diabetic drugs to cancer arise from several meta-analyses of trials not designed to test the hypothesis and observational analytic studies that are subject to bias and confounding factors. Therefore, as the chronology of cancer development is far longer than the time period in which the majority of clinical trials are generally conducted, caution and correct monitoring are essential pending the results of randomised controlled trials of sufficient size and duration in order to minimize the roles of bias.

\section{References}

1. Sun G and Kashyap SR: Cancer risk in type 2 diabetes mellitus: metabolic links and therapeutic considerations. J Nutr Metab 2011: 708183, 2011.

2. Noto H, Goto A, Tsujimoto T, Osame K and Noda M: Latest insights into the risk of cancer in diabetes. J Diabetes Investig 4: 225-232, 2013

3. Habib SL and Rojna M: Diabetes and risk of cancer. ISRN Oncol 2013: 583786, 2013.

4. Giovannucci E, Harlan DM, Archer MC, et al: Diabetes and cancer: a consensus report. Diabetes Care 33: 1674-1685, 2010.

5. Garg SK, Maurer H, Reed K and Selagamsetty R: Diabetes and cancer: two diseases with obesity as a common risk factor. Diabetes Obes Metab 16: 97-110, 2014.

6. Dankner R, Balicer R, Boffetta P, et al: Diabetes, glucose control, glucose lowering medications, and cancer risk: a 10-year population-based historical cohort. BMC Cancer 12: 364, 2012.

7. Lee IM: Physical activity and cancer prevention-data from epidemiologic studies. Med Sci Sports Exerc 35: 1823-1827, 2003.

8. Friedenreich CM, Orenstein MR: Physical activity and cancer prevention: etiologic evidence and biological mechanisms. J Nutr 132: 3456S-3464S, 2002.

9. Chowdhury TA: Diabetes and cancer. QJM 103: 905-915, 2010.

10. Vigneri P, Frasca F, Sciacca L, et al: Diabetes and cancer. Endocr Relat Cancer 16: 1103-1123, 2009.

11. Zendehdel K, Nyren O, Ostenson CG, et al: Cancer incidence in patients with type 1 diabetes mellitus: a population-based cohort study in Sweden. J Natl Cancer Inst 95: 1797-1800, 2003.

12. Szablewski L: Diabetes mellitus: influences on cancer risk. Diabetes Metab Res Rev 30: 543-553, 2014.

13. Handelsman Y, Leroith D, Bloomgarden ZT, et al: Diabetes and cancer - an AACE/ACE consensus statement. Endocr Pract 19: 675-693, 2013.

14. Li C and Kong D: Cancer risks from diabetes therapies: evaluating the evidence. Pharmacol Ther 144: 71-81, 2014.

15. Badrick E and Renehan GA: Diabetes and cancer: 5 years into the recent controversy. Eur J Cancer 50: 2119-2125, 2014.

16. Balbi M, Donadon V, Ghersetti M, et al: Alcohol and HCV chronic infection are risk cofactors of type 2 diabetes mellitus for hepatocellular carcinoma in Italy. Int J Environ Res Public Health 7: 1366-1378, 2010. 
17. Hagberg KW, McGlynn KA, Sahasrabuddhe VV and Jick S Anti-diabetic medications and risk of primary liver cancer in persons with type II diabetes. Br J Cancer 111: 1710-1717, 2014.

18. Burney S, Irfan K, Saif WM and Masud F: Diabetes and pancreatic cancer. JOP 15: 319-321, 2014.

19. Bosetti C, Rosato V, Li D, et al: Diabetes, antidiabetic medications and pancreatic risk: an analysis from the International Pancreatic Cancer Case-Control Consortium. Ann Oncol 25 : 2065-2072, 2014.

20. Li D: Diabetes and pancreatic cancer. Mol Carcinog 51: 64-74, 2012.

21. Stein KB, Snyder CF, Barone BB, et al: Colorectal cancer outcomes, recurrence, and complications in persons with and without diabetes mellitus: a systematic review and meta-analysis. Dig Dis Sci 55: 1839-1851, 2010.

22. Bu WJ, Song L, Zhao DY, Guo B and Liu J: Insulin therapy and the risk of colorectal cancer in patients with type 2 diabetes: a meta-analysis of observational studies. Br J Clin Pharmacol 78: 301-309, 2014.

23. Yin S, Bai H and Jing D: Insulin therapy and colorectal cancer risk among type 2 diabetes mellitus patients: a systemic review and meta-analysis. Diagn Pathol 9: 91, 2014.

24. Peairs KS, Barone BB, Snyder CF, et al: Diabetes mellitus and breast cancer outcomes: a systematic review and meta-analysis. J Clin Oncol 29: 40-46, 2011.
25. Zhu Z, Wang X, Shen Z, et al: Risk of bladder cancer in patients with diabetes mellitus: an updated meta-analysis of 36 observational studies. BMC Cancer 13: 310, 2013.

26. Zhu Z, Zhang X, Shen Z, et al: Diabetes mellitus and risk of bladder cancer: a meta-analysis of cohort studies. PLoS One 8: e56662, 2013.

27. Yoon JM, Son KY, Eom CS, Durrance D and Park SM: Pre-existing diabetes mellitus increases the risk of gastric cancer: a meta-analysis. World J Gastroenterol 19: 936-945, 2013.

28. Tseng CH: Diabetes but not insulin increases the risk of lung cancer: a Taiwanese population-based study. PLoS One 9: e101553, 2014.

29. Gu Y, Wang C, Zheng Y, et al: Cancer incidence and mortality in patients with type 2 diabetes treated with human insulin: a cohort study in Shanghai. PLoS One 8: e53411, 2013.

30. Xu CX, Zhu HH and Zhu YM: Diabetes and cancer: Associations, mechanisms, and implications for medical practice. World J Diabetes 5: 372-380, 2014.

31. Orgel E and Mittelman SD: The links between insulin resistance, diabetes, and cancer. Curr Diab Rep 13: 213-222, 2013. 